\title{
Synthesis and pharmacological trials of new phosphorylated oxazole derivatives antihypertensive properties
}

\author{
Iryna V. Nizhenkovska ${ }^{1}$, Kateryna V. Matskevych ${ }^{1}$, Oleksandr V. Golovchenko ${ }^{2}$, \\ Oksana I. Golovchenko ${ }^{1}$ \\ ${ }^{1}$ Bogomolets National Medical University, T. Shevchenko boulevard 13, 01601, Kyiv, Ukraine \\ ${ }^{2}$ Bioorganic chemistry and petrochemistry institute, Murmanska str. 1, 02094 Kyiv, Ukraine
}

\section{Introduction}

1,3-oxazole derivatives are known to exhibit a wide range of biological effects. They are a part of natural bioactive molecules and synthetic drugs (Harris et al., 2005; Niraimathi et al., 2011). 5amino-1,3-oxazoles are often regarded as masked peptides, so such substances are perspective pharmacophore groups for modification of the peptide chain. In addition, dehydroamino acids are of great interest among biochemists and pharmacologists. Therefore, the synthesis of novel compounds of peptide nature, which contain fragments of dehydroamino acids and 5-amino-1,3oxazole ring, is promising for further pharmacological studies as new bioregulators of various actions.

Phosphorylated oxazole (POD) derivatives are one of the perspective groups of this type of compound with vasodilatation properties. Only sporadic studies of the vasoactive action of this group of compounds in vitro have been identified in the literature, which have not yet been investigated in vivo (Iakovenko et al., 2013).

The above was the basis for pharmacological study of the antihypertensive action of these substances with the prospect of their recommendations for further study and use as medicines for the treatment of hypertension.

\section{Materials and methods}

POD synthesis

POD synthesis was performed using 5-amino-2phthalimidoalkyl-1,3-oxazol-4-ylphosphonic acid diethyl esters.

Screening for vasodilator properties of POD in vitro

Screening of vasodilator properties of a number of PODs and determination of a leader compound was performed on isolated segments of the aorta of rats in vitro (Mikkelsen and Pedersen, 2001). Vascular reactions were recorded using Iris Waveware software for a USB oscilloscope («Iris», США).

Investigation of the antihypertensive effect of $a$ leader compound of $P O D(O V P-1)$ in vivo

The influence of the POD leader compound on blood pressure and hemodynamic parameters was performed on an adrenaline model of acute hypertension with single intravenous (i/v) administration to rabbits of both sexes. The criterion

* matskevychnmu@ukr.net 
for effectiveness was the decrease in mean blood pressure (MBP) after the introduction of OVP-1 and modeling of acute hypertension by not less than 25 $\mathrm{mm} \mathrm{Hg}$ compared to MBP in the blank group (Stephanov, 2001).

\section{Results and discussion}

The possibility of using derivatives of diethyl esters of 5-amino-2-phthalimidoalkyl-1,3-oxazol-4ylphosphonic acids (I) for the synthesis of phosphorylated oxazole derivatives is shown. When exposed to the compounds (I) of hydrazing hydrate, diethyl esters of 2-aminoalkyl-5-alkylamino-1,3oxazol-4-ylphosphonic acids (II) are formed. By the interaction of oxazoles (II) with unsaturated azlactones POD, containing fragments of dehydroamino acids, are synthesized. The developed synthesis method is convenient and preparative, since the transformations take place under mild conditions, which avoids the formation of unwanted side-products, and the POD is obtained in high yields without the use of chromatographic columns.

According to the results of screening studies in vitro, it was found that in isolated segments of the descending part of the thoracic aorta of rats, a compound-leader in a range of phosphorylated oxazole derivatives OVP-1 - OVP-10 was OVP-1, which in concentration $1 \cdot 10^{-5} \mathrm{M}$ most clearly reduced the force amplitude of the adrenalineinitiated $\left(5^{*} 10^{-6} \mathrm{M}\right)$ by $30.9 \%(\mathrm{P}<0.05)$ relative to the baseline.

As a result of in vivo studies, it was found that the criterion for reducing blood pressure in the adrenaline model of acute hypertension was achieved with the introduction of OVP-1 at a dose of $25 \mathrm{mg} / \mathrm{kg}$, which corresponds with the $\mathrm{ED}_{50}$ of the test compound under these conditions of administration $24.19 \pm 2.98 \mathrm{mg} / \mathrm{kg}$ and at a dose of 50 $\mathrm{mg} / \mathrm{kg}\left(2 \mathrm{ED}_{50}\right)$.
It was found that the antihypertensive effect of $25 \mathrm{mg} / \mathrm{kg}$ OVP-1 substance is due to its effect on the reduction of total peripheral vascular resistance by $40.9 \%(\mathrm{P}<0.05)$ relative to baseline and left ventricular working index by $45 \% \quad(\mathrm{P}<$ 0.05) relative to the blank group after adrenaline administration.

\section{Conclusion}

The obtained results are the basis for the purposeful synthesis of potential antihypertensive agents based on phosphorylated oxazole derivatives and for further in-depth preclinical and clinical studies to develop a new original antihypertensive drug to prevent the development and treatment of arterial hypertension.

\section{Conflict of interests: None}

\section{References}

Harris, P., Mui Cheung, Hunter 3rd, R.N., Brown, M.L., Veal, J.M., Nolte, R.T., Wang, L., Liu, W., Crosby, R.M., Johnson, J.H., Epperly, A.H., Kumar, R., Luttrell, D.K., Stafford, J.A., 2005. Discovery and evaluation of 2-anilino-5-arylooxazoles as a novel class VEGFR2 kinase inhibitors. J. Med. Chem. 48, $1610-1619$.

Iakovenko, I.N., Lukashuk, O.I., Kondratyuk, K.M., Golovchenko, A.V., Zhirnov, V.V., Brovarets, V.S., 2013. The synthesis and investigation of vasoactive properties of new phosphorylated peptidomimetics. J. Org. Pharm. Chem., 11 (3), 43-50.

Mikkelsen, E., Pedersen, O.L., 2001. Comparative studies on the effects of tolmesoxide, a tolmesoxide metabolite and nifedipine in isolated blood vessels. Br. J. Pharmacol. 73, 799-805.

Niraimathi, V., Suresh, A.J., Latha, T., 2011. Evaluation of in vitro anti-inflammatory activity of azomethines of aryl oxazoles. E-J Chem. 8, N S1, 392-394.

Stephanov, O.V., 2001. Preclinical studies of medicines. Avicena, 527. 